\title{
Dual Composite Right/Left-Handed Coplanar Waveguide Transmission Line using Inductively Connected Split-Ring Resonators
}

\author{
Angel Belenguer, Member, IEEE, Joaquin Cascon, Alejandro L. Borja, Héctor Esteban, Member, IEEE, \\ and Vicente E. Boria, Senior Member, IEEE
}

\begin{abstract}
In this paper a simple geometrical modification of the well-known split-ring resonator (SRR) topology is proposed. This modification consists on joining the opposite sides of the rings by means of an inductive connection. Due to this modification, it is possible to synthesize a dual composite right/left handed artificial transmission line from a host coplanar waveguide (CPW). In addition, it is quite straightforward to synthesize a dual balanced line showing a wider transmission bandwidth (around $13 \%$ for $3 \mathrm{GHz}$ and $8 \%$ for $6 \mathrm{GHz}$ ) with this new SRR geometry. In fact, in order to design a balanced line, one must just modify the inductance of the direct connection between the rings, which can be derived from the equivalent circuit of this new ring, also presented in this paper. The final result is a balanced artificial CPW transmission line with a dual composite right/lefthanded behavior. In this case, the right-handed transmission band is placed below the left-handed one, and there is no frequency separation between both transmission bands.
\end{abstract}

Index Terms-Double-negative materials, left-handed (LH) transmission lines, metamaterials, composite right/left-handed (CRLH), dual composite right/left-handed (D-CRLH), Coplanar waveguide (CPW), Inductively Connected Split Ring Resonator (IC-SRR)

\section{INTRODUCTION}

$\mathbf{M}$ ANY research efforts have been devoted to synthesize a left-handed propagating medium from a conventional transmission line, usually, a microstrip line or a coplanar waveguide (CPW) line. The left-handed behavior is obtained when the host transmission line is either loaded with splitring resonators (SRRs) [1]-[3] or their dual counterpart, the complementary split-ring resonator (CSRR) [4]-[7], as well as with reactive elements [8], [9]. The result is a composite right/left-handed transmission line (CRLH) with a left-handed propagation band and one or more right-handed bands at higher frequencies, since the background medium is itself a right-handed transmission line.

Due to the resonant nature of SRR and CSRR, special efforts have been made for the synthesis of artificial transmission lines

This work was supported by the Ministerio de Ciencia e Innovación, Spanish Goverment, under Research Projects TEC2010-21520-C04-03 and -01 , and by the Autonomous Government of Castilla-La Mancha under Research Projects PPII10-0047-0220 and PPII10-0027-1277.

A. Belenguer, J. Cascon, and A. L. Borja are with Departamento de Ingeniería eléctrica, electrónica, automática y comunicaciones, Universidad de Castilla-La Mancha, Escuela Politécnica de Cuenca, Campus Universitario, 16071 Cuenca, Spain (e-mail: angel.belenguer@uclm.es; joaquin.cascon@uclm.es; alejandro.lucas@uclm.es).

H. Esteban and V. E. Boria are with Departamento de Comunicaciones, Universidad Politécnica de Valencia, 46022 Valencia, Spain (e-mail: hesteban@dcom.upv.es; vboria@dcom.upv.es). exhibiting improved (i.e., wider) bandwidths. A broadband response can be obtained when the right- and left-handed behavior coincide. A balanced line is synthesized in this case. For example, in [10], the dimensions of the interdigital capacitor and the shunt short-circuited stub of [8] are modified to obtain a balanced line. Likewise, in [5], the gap capacitor of [4] is replaced by an interdigital capacitor to balance the line. Finally, in [11], the basic cell of [2] is modified by adding shunt open-circuited stubs and an interdigital capacitor to achieve the same goal.

Moreover, in 2006, Caloz presented [12] a theoretical study of a structure that shows a dual behavior when compared with a CRLH line. This new structure exhibits its left-handed band at high frequencies, and its right-handed band at low frequencies. Several implementations of this theoretical structure have been reported [13]-[16]. These preliminary studies show that this new structure introduces lower losses and provides larger bandwidths than its dual counterpart [15].

In this paper, a geometrical modification for the SRR is proposed. The opposite sides of the rings are directly connected with an inductive load (see Fig. 9). In addition, this inductively connected SRRs (IC-SRRs) are united to the CPW by metal vias (see Fig. 11), so that the interaction with the line is also modified in comparison to other previously published proposals [2], [11]. Because of these simple modifications, the new CPW loaded line behaves as a Dual CLRH (D-CLRH) line. Besides, this new line can be balanced quite easily by appropriately adjusting the inductance of the connection between the two rings.

The paper is organized as follows. Section II discusses the derivation of equivalent circuits for SRR-based particles. Section III reviews the equivalent circuit implementation with the new proposed IC-SRR particle. Section IV presents two specific designs of novel artificial transmission lines based on such IC-SRR particles, and their related results. In Section $\mathrm{V}$ fabrication issues related to these novel structures are discussed, and they are taken into account to re-design the artificial transmission lines of section IV. Finally, the main conclusions of the study are outlined in Section VI.

\section{EQUiVALENT CIRCUIT}

The starting point for the equivalent circuits derivations is the original circuit from [17], [18] shown in Fig. 2. This circuit describes the behavior of a CPW coupled with two 


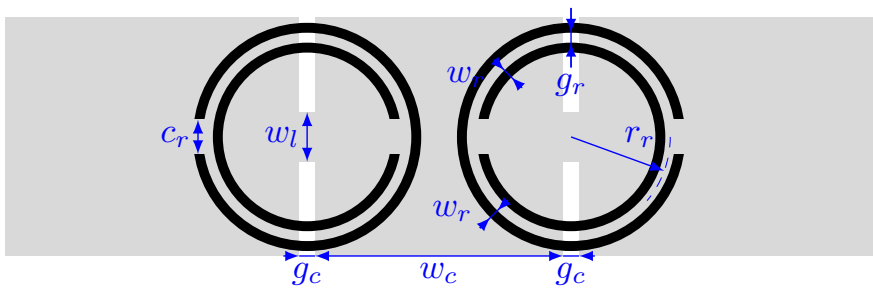

Fig. 1. Basic cell. Gray is top layer; black is bottom layer.
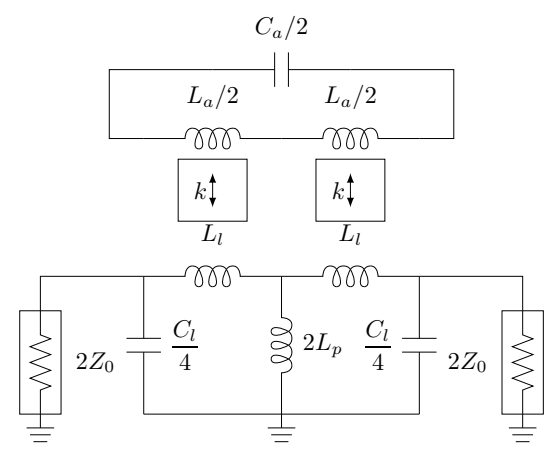

Fig. 2. Equivalent circuit for the SRR-based basic cell of [17], [18].

SRRs, and loaded with two parallel strips that connect the ground planes with the central line (see Fig. 1).

The equivalent circuit of a SRR is deduced from its physical behavior [1], and then it is simplified to an LC resonator (see Fig. 2). However, in order to modify the SRR, this simplified equivalent circuit is not useful. A circuit model closer to the physical phenomena that give rise to the SRR resonance is needed.

The physical process behind the SRR resonance is explicitly shown in Fig. 3. First, the current flows along the first ring. Since there is a capacitive coupling between the rings, this current is gradually transferred to the second ring. Then, when the current reaches the cut in the first ring, it entirely flows along the second one, and there, the process is exactly the opposite. The current flows along the second ring and is progressively transferred to the first ring. When it reaches the cut in the second ring it entirely flows, again, along the first one, just as it was at the beginning of the process. Fig. 3 shows an equivalent circuit that fully describes this physical behavior.

It can be noted that this new model is completely equivalent to the equivalent circuit shown in Fig. 2. In fact, the circuits

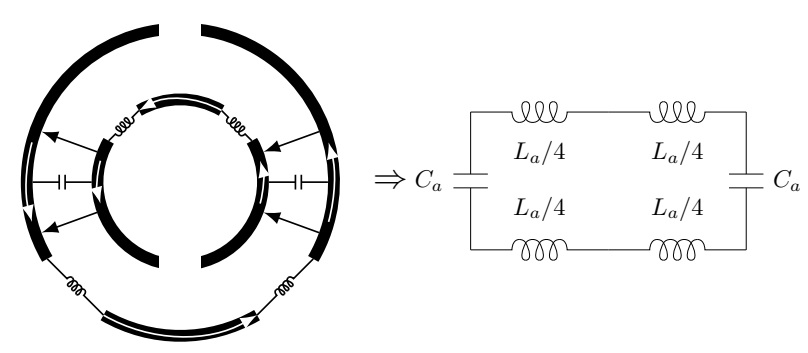

Fig. 3. Ring equivalent circuit.
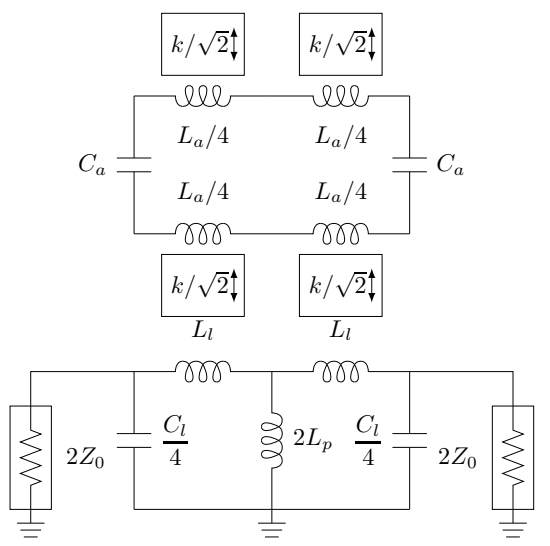

Fig. 4. New equivalent circuit with explicitly separated rings.

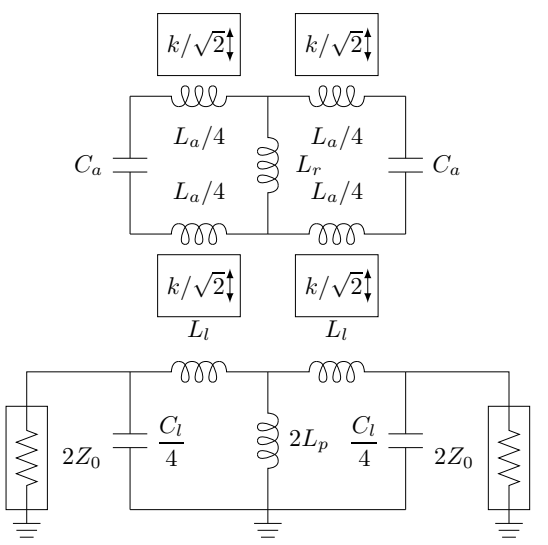

Fig. 5. Equivalent circuit with direct connection between the SRR rings.

of Fig. 2 and Fig. 4 are the same ones if the circuit elements of the ring in Fig. 4 are adequately grouped.

Since the contribution of each individual SRR ring is explicitly considered, it is possible to study what happens, for example, when the rings are directly connected using an inductance. An equivalent circuit that models this direct connection is shown in Fig. 5.

Then, in order to analyze the influence of this new inductance ( $L_{r}$ in Fig. 5), a specific SRR-based basic cell, like the one shown in Fig. 1, has been designed.

This cell has been implemented on a Rogers $4003 \mathrm{C}^{\mathrm{TM}}$ substrate $\left(\epsilon_{r}=3.55\right.$ and $\left.\tan (\delta)=0.0027\right)$ of $1.524 \mathrm{~mm}$ thick and $35 \mu \mathrm{m}$ of copper metallization, and designed to show a left-handed transmission band around $3 \mathrm{GHz}$. In order to obtain the dimensions and equivalent circuit elements of this cell, the following design procedure has been followed,

1) The ring strip width and gap are fixed, so that they can be fabricated with the milling machine used in the fabrication process.

Since one of the main goals is to design a very compact unit cell (as much small as possible), a reasonably short gap should be selected. This provides a quite high ring capacitance and, therefore, a lower inductance is needed for achieving the desired resonant frequency. Besides, since the ring shows a serial resonance (see Fig. 3), the 
TABLE I

EQUIVALENT CIRCUIT ELEMENTS OF THE SRR LOADED CPW.

\begin{tabular}{lll} 
& $3 \mathrm{GHz}$ & $6 \mathrm{GHz}$ \\
\hline$L_{l}$ & $1.75 \mathrm{nH}$ & $1 \mathrm{nH}$ \\
$C_{l}$ & $0.637 \mathrm{pF}$ & $0.32 \mathrm{pF}$ \\
$l_{l}$ & $2 r_{r}$ & $2 r_{r}$ \\
$L_{p}$ & $0.2 \mathrm{nH}$ & $0.2 \mathrm{nH}$ \\
$L_{a}$ & $17.65 \mathrm{nH}$ & $8.365 \mathrm{nH}$ \\
$C_{a}$ & $0.318 \mathrm{pF}$ & $0.181 \mathrm{pF}$ \\
$k$ & 0.26 & 0.169 \\
\hline
\end{tabular}

TABLE II

BASIC CELL DIMENSIONS OF THE SRR LOADED CPW.

\begin{tabular}{lll} 
& $3 \mathrm{GHz}$ & $6 \mathrm{GHz}$ \\
\hline$w_{c}$ & $14 \mathrm{~mm}$ & $8 \mathrm{~mm}$ \\
$g_{c}$ & $0.78 \mathrm{~mm}$ & $0.57 \mathrm{~mm}$ \\
$r_{r}$ & $3.825 \mathrm{~mm}$ & $1.95 \mathrm{~mm}$ \\
$c_{r}$ & $1.6 \mathrm{~mm}$ & $0.8 \mathrm{~mm}$ \\
$w_{r}$ & $0.4 \mathrm{~mm}$ & $0.4 \mathrm{~mm}$ \\
$g_{r}$ & $0.4 \mathrm{~mm}$ & $0.4 \mathrm{~mm}$ \\
$w_{l}$ & $1 \mathrm{~mm}$ & $1 \mathrm{~mm}$ \\
\hline
\end{tabular}

lower the inductance and the higher the capacitance, the wider the bandwidth. For both reasons, size and bandwidth, a shorter gap (physically feasible) is a good starting point.

Therefore, and considering the characteristics of the milling machine, a gap of $0.4 \mathrm{~mm}$ is selected.

2) By applying the expressions of [1], and the aforementioned geometrical constraints, the ring radius is calculated and also its equivalent inductance and capacitance.

3) The line is now designed. Since the ring radius is known, a sufficiently wide coplanar strip must be selected, for example a $14 \mathrm{~mm}$ wide strip. Then, applying the design formulas of [19], the dimmensions of a $50 \Omega \mathrm{CPW}$ are found.

4) By using these dimensions, an initial cell is simulated using a commercial software, Ansoft HFSS ${ }^{\mathrm{TM}}$. Since all the expressions used to calculate the equivalent circuit parameters are approximate, the cell response is slightly frequency-shifted. Then, by adjusting the ring radius, the resonant frequency can be translated to its desired value.

5) New equivalent circuit values are calculated by optimization in order to obtain a response as close as possible to the response of the tuned cell.

Following this procedure, a $6 \mathrm{GHz}$ cell has also been designed. The values of the equivalent circuit elements are shown in Table I. $C_{l}$ and $L_{l}$ can be calculated by multiplying the per-unit-length inductance and capacitance of the CPW by the line length, $l_{l}$. The rest of the parameters are computed as indicated in [1].

The physical dimensions corresponding to these equivalent circuits (see Fig. 1) are shown in Table II

The values in the first column of Table I have been applied to the equivalent circuit of Fig. 5. Later, in order to evaluate the effect of a direct connection between rings, this equivalent circuit has been analyzed using different $L_{r}$ values. The results of this analysis are shown in Fig. 6. It can be seen how the original passband, which is around $3 \mathrm{GHz}$, is unaffected when

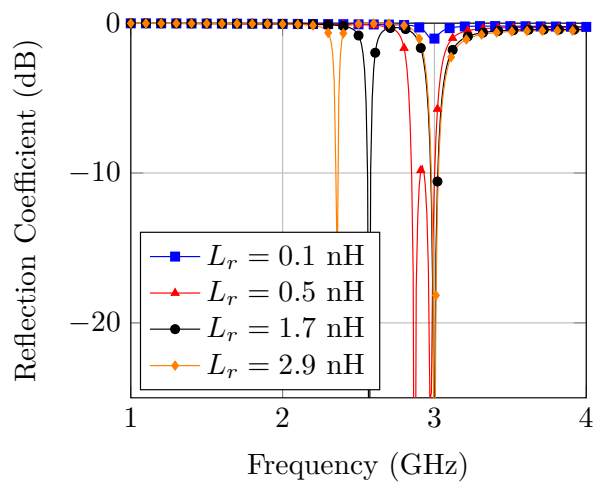

Fig. 6. Parametric analysis of the equivalent circuit of Fig. 5.

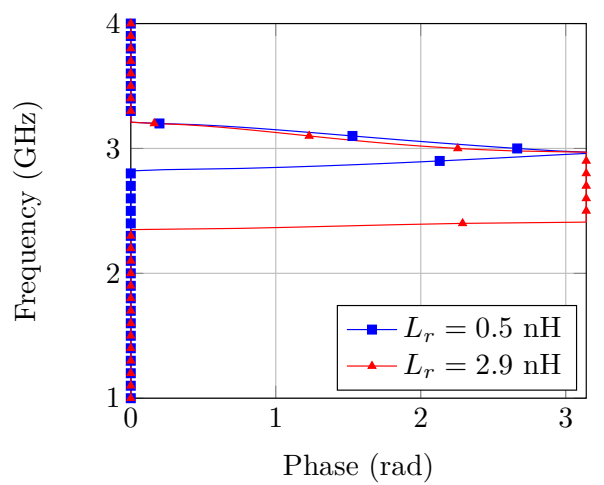

Fig. 7. Dispersion diagram for $L_{r}=0.5 \mathrm{nH}$ and $L_{r}=2.9 \mathrm{nH}$.

$L_{r}$ is modified. However, a second passband appears because of this connecting inductance. As $L_{r}$ is increased, the central frequency of this band decreases, whereas the original band is hardly modified. Only when the connecting inductance value is really small, the original passband is affected. In this case the rings are short-circuited and neither of the two bands exists.

The original passband is a left-handed transmission band. On the other hand, the new band at lower frequencies is righthanded. Therefore, the result is a D-CRLH line. This fact can be confirmed observing the cell dispersion diagram, shown in Fig. 7, and computed using the following equation [20],

$$
\beta l_{l}=\cos ^{-1}\left(\frac{1-s_{11} s_{22}+s_{12} s_{21}}{2 s_{21}}\right)
$$

\section{IC-SRR IMPLEMENTATION}

In order to implement a real device showing nearly the same behaviour as the equivalent circuit in Fig. 5, the SRR rings must be connected using an inductance, as shown in Fig. 8 .

By doing this, a new resonator is obtained, which is the proposed IC-SRR. This new composite ring behaves as the resonator of the equivalent circuit in Fig. 5.

Then, applying the new equivalent circuit of Fig. 5 and using the commercial software Ansoft $\mathrm{HFSS}^{\mathrm{TM}}$, a basic cell on Rogers $4003 \mathrm{C}^{\mathrm{TM}}$ substrate $\left(\epsilon_{r}=3.55\right.$ and $\left.\tan (\delta)=0.0027\right)$ of $1.524 \mathrm{~mm}$ thick and $35 \mu \mathrm{m}$ of copper metallization has been designed and tuned at $3 \mathrm{GHz}$. The design procedure is exactly the same followed to obtain a basic cell. The only difference 


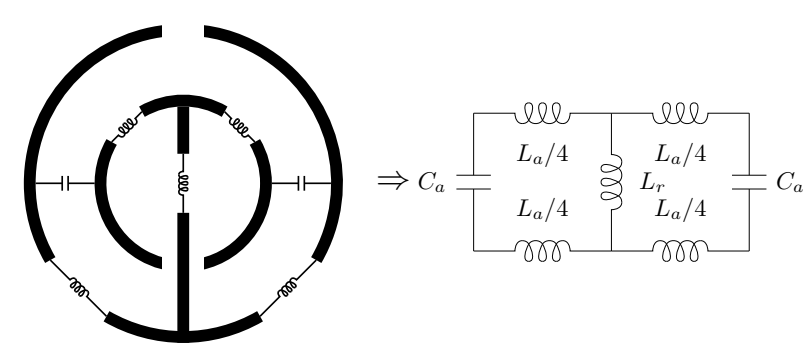

Fig. 8. IC-SRR and equivalent circuit.

TABLE III

NON-CONNECTED IC-SRR CELL DIMENSIONS AND CIRCUIT ELEMENTS.

\begin{tabular}{ll|ll}
\multicolumn{2}{c|}{ Dimensions } & \multicolumn{2}{c}{ Circuit elements } \\
\hline$w_{c}$ & $14 \mathrm{~mm}$ & $L_{l}$ & $2.12 \mathrm{nH}$ \\
$g_{c}$ & $0.78 \mathrm{~mm}$ & $C_{l}$ & $0.77 \mathrm{pF}$ \\
$r_{r}$ & $4.65 \mathrm{~mm}$ & $l_{l}$ & $2 r_{r}$ \\
$c_{r}$ & $4.6 \mathrm{~mm}$ & $L_{p}$ & $0.2 \mathrm{nH}$ \\
$w_{r}$ & $0.4 \mathrm{~mm}$ & $L_{a}$ & $18.44 \mathrm{nH}$ \\
$g_{r}$ & $0.4 \mathrm{~mm}$ & $C_{a}$ & $0.291 \mathrm{pF}$ \\
$w_{l}$ & $1 \mathrm{~mm}$ & $k$ & 0.242 \\
$w_{b}$ & $3.8 \mathrm{~mm}$ & $L_{r}$ & $0.58 \mathrm{nH}$ \\
\hline
\end{tabular}

appears in the tuning step. Now, in order to tune the response of the cell, the ring radius and the width of the new connecting strip are adjusted, while in the basic cell tuning, only the ring radius was modified. The final dimensions and circuit elements (see Fig. 5) of this new IC-SRR cell are shown in Table III, and its geometry can be seen in Fig. 9.

Finally, Fig. 10 shows the results for this cell. The results given by the commercial software HFSS $^{\mathrm{TM}}$ are significantly different from the results given by the equivalent circuit of Fig. 5, above the resonances, because the parasitic capacitance connecting the SRR rings through the host CPW is not considered in the equivalent circuit of Fig. 5. This parasitic capacitance gives rise to an additional transmission band at higher frequencies (see Fig. 10). This is the reason why $\mathrm{HFSS}^{\mathrm{TM}}$ results give a notably asymmetric response. The simulated results show that this asymmetry is not fully modeled with the equivalent circuit of Fig. 5 and requires proper treatment, since the aforementioned transmission band is not considered in this case.

Besides, a design at $6 \mathrm{GHz}$ is not possible with this configuration. In this case, the inductance value required to connect the two rings is too small (i.e. $L_{r} \approx 0.2 \mathrm{nH}$ ), which involves a very wide connecting strip (i.e. $w_{b}>4 \mathrm{~mm}$ ), thus making the cell physically non-realizable.

In order to eliminate this undesired and uncontrolled transmission band at high frequencies, the parasitic capacitance that connects the rings through the host CPW has to be cancelled. To accomplish this objective, the rings have been directly connected to the CPW using plated via holes (see Fig. 11).

This direct connection between the rings and the host CPW has an additional positive effect. It has been demonstrated before that the inductance of the connecting strip required to synthesize this kind of cell is quite low, i.e. the strip connecting the rings must be quite wide (see Table III). Unfortunately,

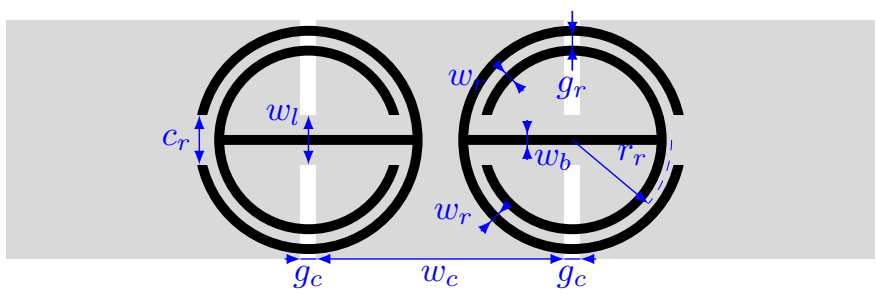

Fig. 9. Non-connected IC-SRR cell. Gray is top layer; black is bottom layer.

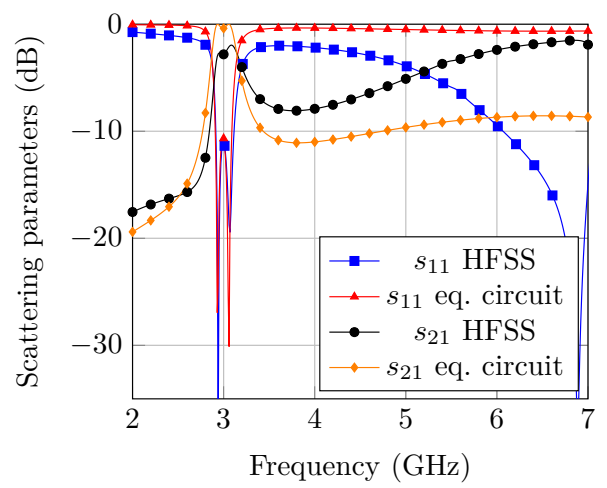

Fig. 10. Non-connected IC-SRR cell (Ansoft HFSS ${ }^{\mathrm{TM}}$ ) vs. equivalent circuit.

such a wide connection blocks the magnetic field from the CPW. As a result, the coupling between line and ring is reduced, and, therefore, the cell bandwidth decreases [1]. Even the required width can be so wide that the cell can become physically non-realizable. Fortunately, if the rings are connected to the CPW, a shunt inductance appears at the terminals of the direct connection. In this case, the direct inductance can be higher and, therefore, the width of the connecting strip will be narrower. Then, the coupling level increases and, as a result, bandwidth increases as well. Besides, with this configuration, it has been possible to design a $6 \mathrm{GHz}$ cell.

However, the circuit of Fig. 5 is not able to accurately describe the behavior of this cell, because it completely ignores the CPW-to-SRR direct connection. Therefore, to appropriately model this new direct connection, the circuit of Fig. 5 has been slightly modified. The via holes have been replaced by inductances (see Fig. 12). These inductances have been computed by using the empirical formula of [21].

Fig. 13 shows the simulated $\left(\mathrm{HFSS}^{\mathrm{TM}}\right)$ scattering parameters for the $3 \mathrm{GHz}$ and $6 \mathrm{GHz}$ cells. In this case, since the parasitic capacitance has been cancelled, the results are quite similar to the results given by the final equivalent circuit of Fig. 12. Here, the non-desired transmission band of Fig. 10 disappears, and a more symmetric response is obtained, as expected.

Finally, the values of the equivalent circuit elements for these cells can be seen in Table IV, and the physical dimensions in Table V.

\section{REsults}

\section{A. First designs}

The previously designed cells have been fabricated and measured. In order to connect the cells to the vector network 


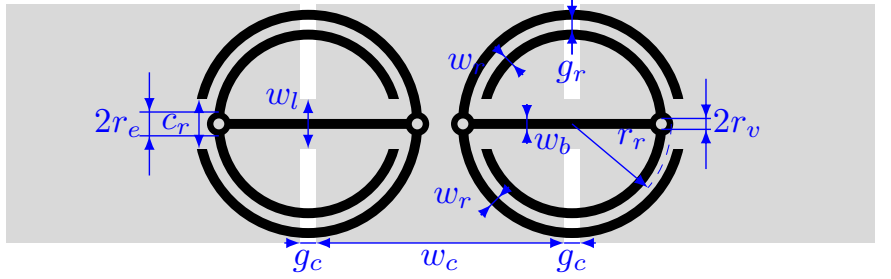

Fig. 11. Connected IC-SRR cell. Gray is top layer; black is bottom layer.

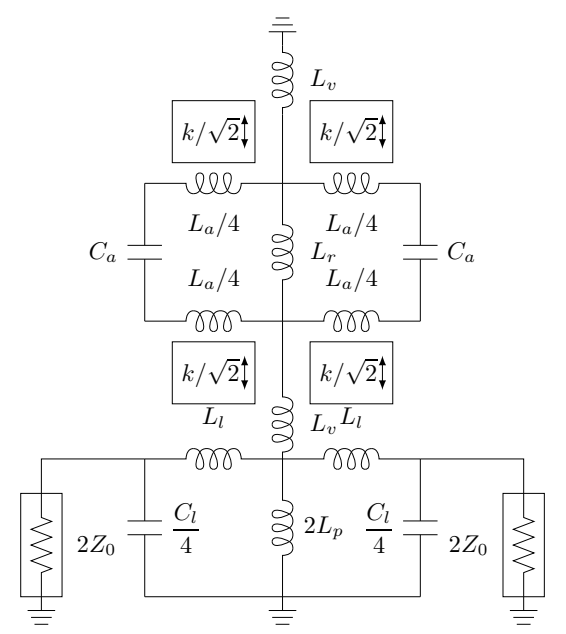

Fig. 12. Equivalent circuit of the new connected IC-SRR cell.

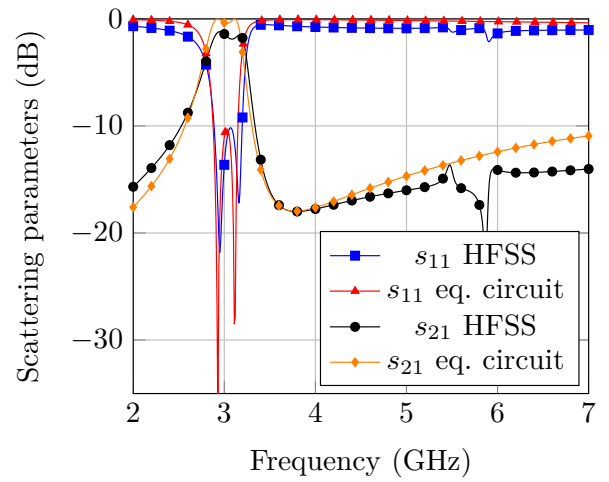

(a) $3 \mathrm{GHz}$

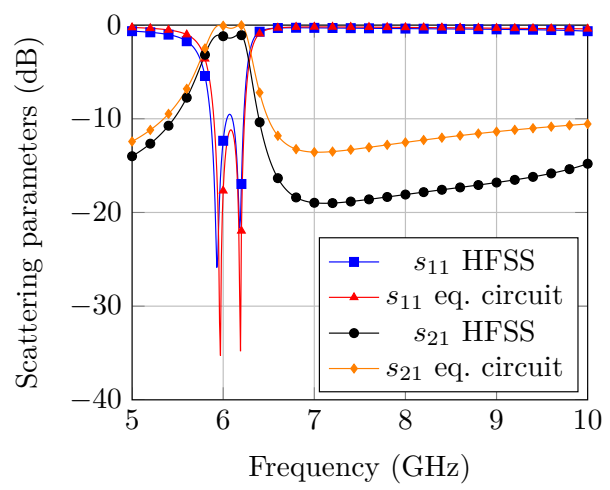

(b) $6 \mathrm{GHz}$

Fig. 13. Connected IC-SRR cell (Ansoft HFSS ${ }^{\mathrm{TM}}$ ) vs. equivalent circuit results.
TABLE IV

EQUIVALENT CIRCUIT ELEMENTS OF THE CONNECTED IC-SRR LOADED CPW.

\begin{tabular}{lll} 
& $3 \mathrm{GHz}$ & $6 \mathrm{GHz}$ \\
\hline$L_{l}$ & $1.75 \mathrm{nH}$ & $1.17 \mathrm{nH}$ \\
$C_{l}$ & $0.637 \mathrm{pF}$ & $0.374 \mathrm{pF}$ \\
$l_{l}$ & $2 r_{r}$ & $2 r_{r}$ \\
$g_{r}$ & $0.4 \mathrm{~mm}$ & $0.4 \mathrm{~mm}$ \\
$L_{p}$ & $0.2 \mathrm{nH}$ & $0.2 \mathrm{nH}$ \\
$L_{a}$ & $16.74 \mathrm{nH}$ & $8.57 \mathrm{nH}$ \\
$C_{a}$ & $0.304 \mathrm{pF}$ & $0.153 \mathrm{pF}$ \\
$k$ & 0.265 & 0.188 \\
$L_{r}$ & $7.98 \mathrm{nH}$ & $0.371 \mathrm{nH}$ \\
$L_{v}$ & $0.261 \mathrm{nH}$ & $0.207 \mathrm{nH}$ \\
\hline
\end{tabular}

TABLE V

BASIC CELL DIMENSIONS OF THE CONNECTED IC-SRR LOADED CPW.

\begin{tabular}{lll} 
& $3 \mathrm{GHz}$ & $6 \mathrm{GHz}$ \\
\hline$w_{c}$ & $14 \mathrm{~mm}$ & $8 \mathrm{~mm}$ \\
$g_{c}$ & $0.78 \mathrm{~mm}$ & $0.57 \mathrm{~mm}$ \\
$r_{r}$ & $3.835 \mathrm{~mm}$ & $2.28 \mathrm{~mm}$ \\
$c_{r}$ & $2.4 \mathrm{~mm}$ & $2.4 \mathrm{~mm}$ \\
$w_{r}$ & $0.4 \mathrm{~mm}$ & $0.4 \mathrm{~mm}$ \\
$g_{r}$ & $0.4 \mathrm{~mm}$ & $0.4 \mathrm{~mm}$ \\
$w_{l}$ & $1 \mathrm{~mm}$ & $1 \mathrm{~mm}$ \\
$w_{b}$ & $0.1 \mathrm{~mm}$ & $1.4 \mathrm{~mm}$ \\
$r_{e}$ & $0.8 \mathrm{~mm}$ & $0.8 \mathrm{~mm}$ \\
$r_{v}$ & $0.5 \mathrm{~mm}$ & $0.5 \mathrm{~mm}$ \\
\hline
\end{tabular}

analyzer, a taper to a narrower CPW has been added, so that a SMA connector can be soldered at the device ports (see Fig. 15). For both designs, the input/output CPW line has a gap dimension of $0.44 \mathrm{~mm}$ and a central strip of $5 \mathrm{~mm}$. The taper is a linear transition from this narrow line to the different, and always wider, CPW lines of the cells (see again Fig. 15).

S-parameter measurements of these prototypes can be seen in Fig. 14. These results are compared with the HFSS $^{\mathrm{TM}}$ data. These last results slightly differ from those shown at previous sections because, in this case, the devices have been simulated considering the tapers, the narrower feeding lines and the SMA connectors. The passband is preserved (same frequency and bandwidth) and the insertion loss increases but remains acceptable.

The transmission bandwidth achieved with these prototypes is notably higher, with a fractional bandwidth of $13 \%$ for the $3 \mathrm{GHz}$ cell and $8 \%$ for the $6 \mathrm{GHz}$ cell, in comparison to the bandwidth provided by the basic cell of Fig. 1, which is typically around 2\% [22] for a single cell and a reasonably high rejection outside the transmission band.

Besides, the fabricated prototypes present a moderate/low insertion loss level in the transmission band. The insertion loss is, for both designs, below $2 \mathrm{~dB}$, even considering the contribution of tapers and SMA connectors.

It can be seen that measurements present a noticeable frequency shift when compared with simulations. This is due to the fabrication process. To fabricate these prototypes, a milling machine has been employed. It is very difficult to exactly control the milling depth when milling a circuit. By analyzing the fabricated prototypes, an overmilling of $100 \mu \mathrm{m}$ has been estimated. This overmilling, not considered when designing these prototypes, is the main responsible of this 


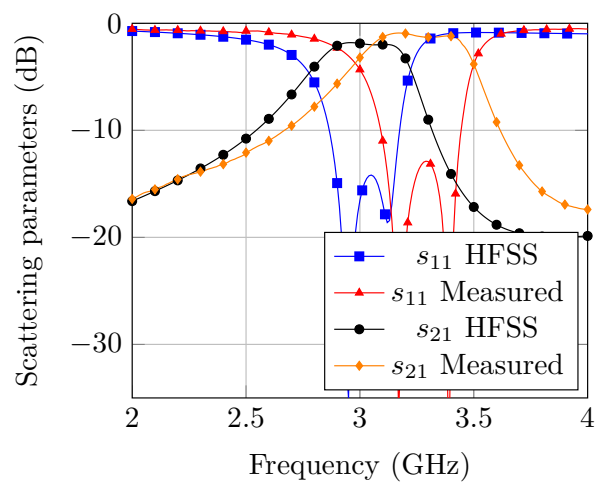

(a) $3 \mathrm{GHz}$

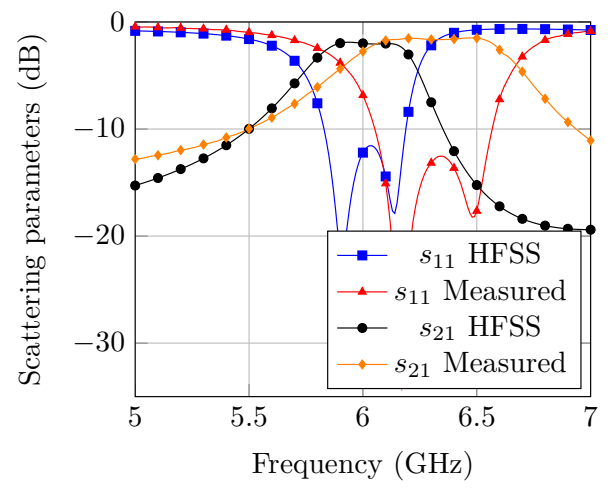

(b) $6 \mathrm{GHz}$

Fig. 14. Comparison between measurements and simulated results of the connected IC-SRR cell (overmilling not considered).

TABLE VI

BASIC CELL DIMENSIONS OF THE CONNECTED IC-SRR LOADED CPW CONSIDERING OVERMILLING.

\begin{tabular}{lll} 
& $3 \mathrm{GHz}$ & $6 \mathrm{GHz}$ \\
\hline$w_{c}$ & $14 \mathrm{~mm}$ & $8 \mathrm{~mm}$ \\
$g_{c}$ & $0.78 \mathrm{~mm}$ & $0.57 \mathrm{~mm}$ \\
$r_{r}$ & $4.11 \mathrm{~mm}$ & $2.43 \mathrm{~mm}$ \\
$c_{r}$ & $2.4 \mathrm{~mm}$ & $2.4 \mathrm{~mm}$ \\
$w_{r}$ & $0.4 \mathrm{~mm}$ & $0.4 \mathrm{~mm}$ \\
$g_{r}$ & $0.4 \mathrm{~mm}$ & $0.4 \mathrm{~mm}$ \\
$w_{l}$ & $1 \mathrm{~mm}$ & $1 \mathrm{~mm}$ \\
$w_{b}$ & $0.1 \mathrm{~mm}$ & $1.4 \mathrm{~mm}$ \\
$r_{e}$ & $0.8 \mathrm{~mm}$ & $0.8 \mathrm{~mm}$ \\
$r_{v}$ & $0.5 \mathrm{~mm}$ & $0.5 \mathrm{~mm}$ \\
\hline
\end{tabular}

undesired frequency shift.

\section{B. Overmilling}

In order to confirm that this frequency shift is caused by overmilling, the cells have been redesigned considering an overmilling of $100 \mu \mathrm{m}$ in the HFSS ${ }^{\mathrm{TM}}$ simulations. The dimensions of these modified cells are shown in Table VI.

These corrected cells have been fabricated (see Fig. 15). The comparison of simulations and measurements for these new prototypes is shown in Fig. 16. These new results confirm that the undesired frequency shift was indeed caused by overmilling. Results for these corrected cells have a much lower frequency shift than first designs. Even so, it is impossible to exactly determine the actual overmilling of every fabrication. Besides, there are other error sources that cannot be controlled,

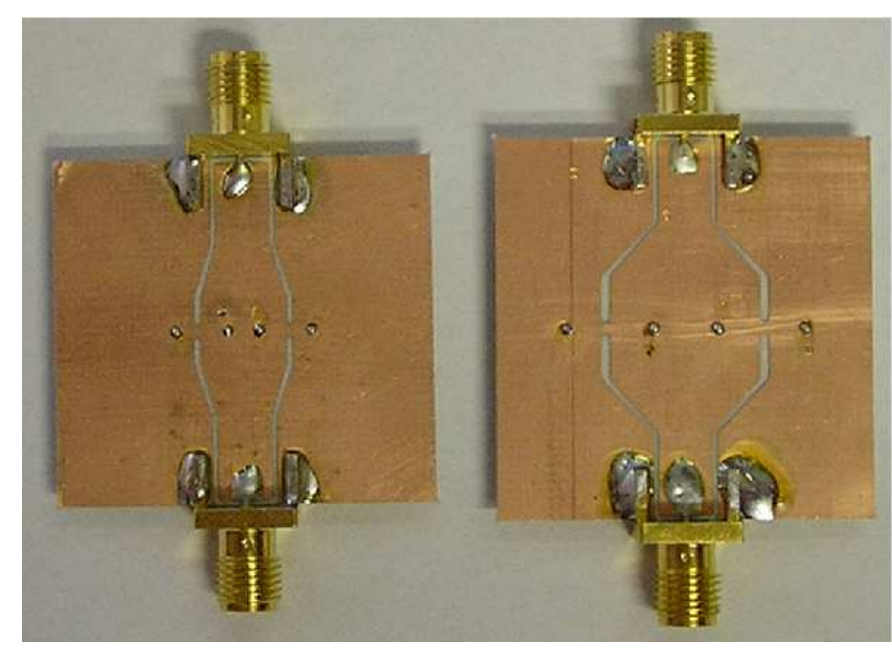

(a) Top

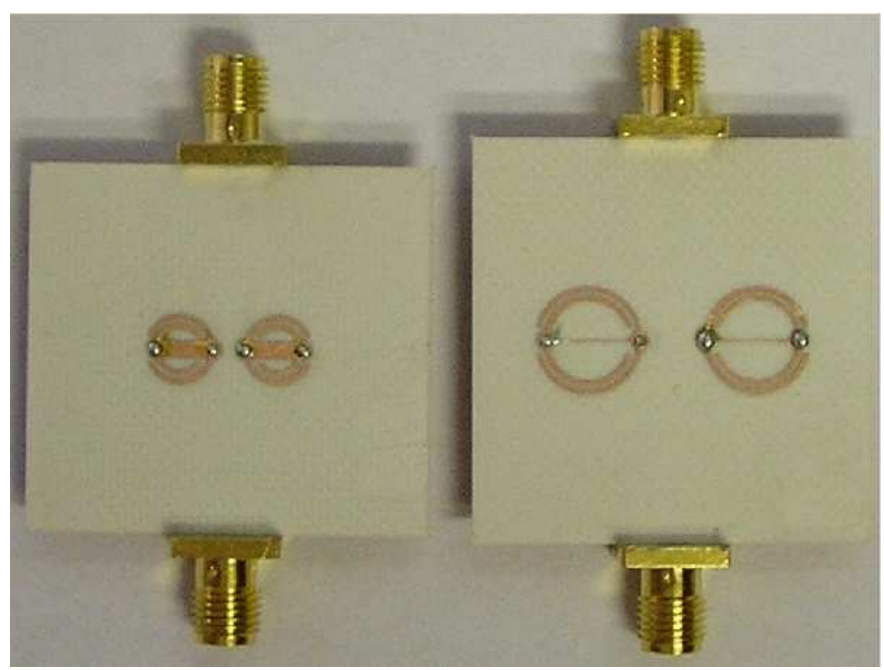

(b) Bottom

Fig. 15. Photograph of two fabricated IC-SRR prototypes designed to work at $3 \mathrm{GHz}$ and $6 \mathrm{GHz}$.

mainly machining tolerances and slight deviations, from their nominal values, of the substrate geometrical and electrical characteristics. This is the reason why these results are still slightly shifted.

Finally, Fig. 17 shows the dispersion diagrams and retrieved effective $\epsilon$ and $\mu$ for the last prototypes with overmilling correction. The dispersion diagrams have been computed, again, using (1). The effective constants, $\epsilon$ and $\mu$, for an infinite cascade of unitary cells, have been calculated applying [23]. In these last calculations, a cascading period of $19 \mathrm{~mm}$ has been considered for the $3 \mathrm{GHz}$ prototype. Similarly, a $9.5 \mathrm{~mm}$ repetition period has been used for the cascade of $6 \mathrm{GHz}$ cells. It can be observed that a right-handed propagation is achieved in the lower frequencies of the transmission band (phase augments as frequency increases and $\epsilon>0, \mu>0$ ) followed by a left-handed transmission band (phase diminishes as frequency increases and $\epsilon<0, \mu<0$ ). These results confirm that, because of the new proposed IC-SRRs, a DCLRH line has been successfully designed for 3 and $6 \mathrm{GHz}$. 


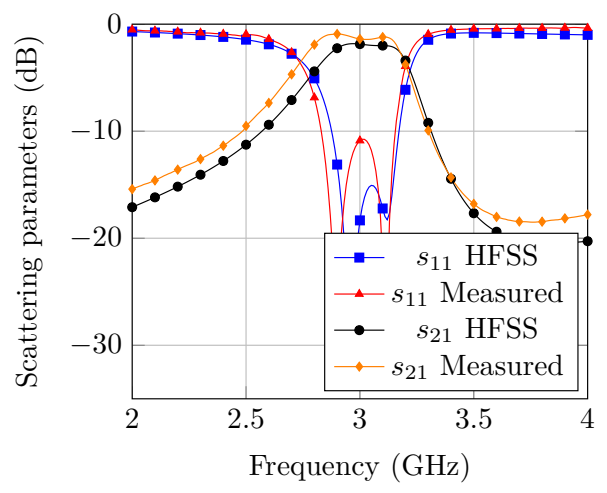

(a) $3 \mathrm{GHz}$

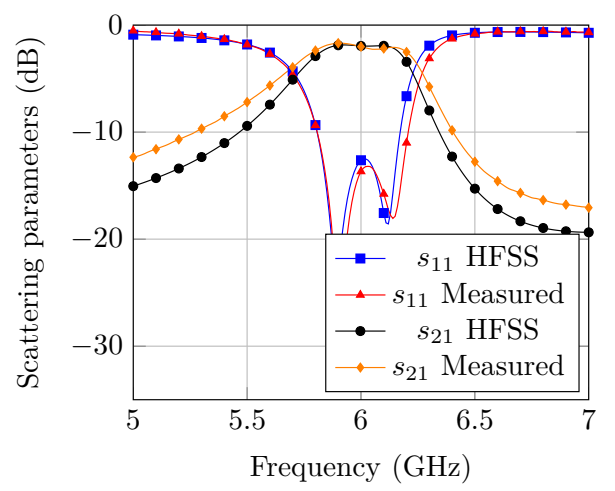

(b) $6 \mathrm{GHz}$

Fig. 16. Comparison between measurements and simulated results of the connected IC-SRR cell (overmilling considered).

\section{CONCLUSions}

In this paper, a new cell for synthesizing D-CLRH lines has been successfully presented. This cell has been implemented by loading a CPW with IC-SRRs, which are obtained by modifying the conventional SRR geometry. In this new particle, opposite sides of both SRR rings are directly joined by means of an inductive connection.

The IC-SRR-based cell is straightforward to balance and, therefore, it is possible to achieve wider passbands. In fact, a percentual bandwidth of $13 \%$ has been achieved for the $3 \mathrm{GHz}$ cell and of $8 \%$ for the $6 \mathrm{GHz}$ cell. Previously, in order to balance these kind of cells, several strategies have been used. Most of these proposals modify the background line, in our case a host CPW. This line is loaded with several reactive elements [4], [5], [8], [10], [11], so that the left and right-handed behavior converge around the same frequency. In this paper, the strategy is completely the opposite. Instead of modifying the background line, the resonant element is modified. As a result a new resonator emerges, the IC-SRR. By using these modified rings, balancing a cell is quite easy, since one has simply to appropriately change the IC-SRR inductive connection.

Besides, the fabricated prototypes show low/moderate insertion loss (below 2dB including tapers and SMA connectors). In fact, this is one of the main advantages of D-CLRH lines.

Finally, it is very important to remark that the new cell takes up the same area as the original one. Therefore, bandwidth has been increased (balanced cell) and losses reduced (dual line)

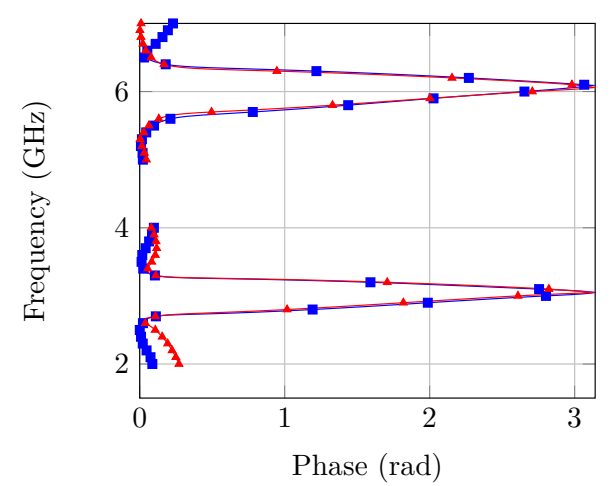

(a) Dispersion diagram

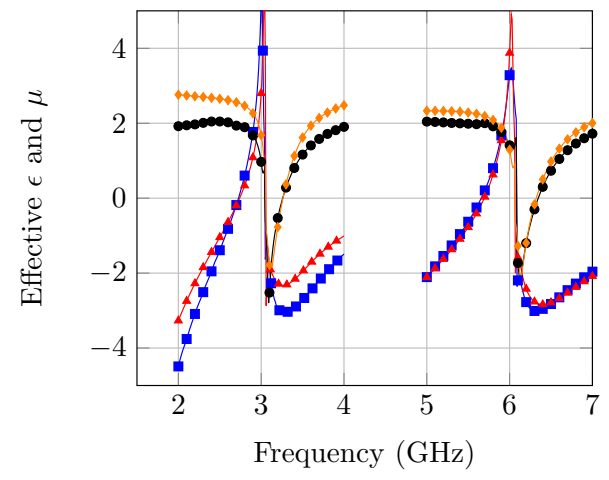

(b) Effective $\epsilon$ and $\mu$

Fig. 17. (a) Dispersion diagram for the fabricated prototypes: measurements (blue line with squared marks) vs. HFSS ${ }^{\mathrm{TM}}$ (red line with triangular marks). (b) Effective $\epsilon$ : measurements (blue line with squared marks) $\mathrm{HFSS}^{\mathrm{TM}}$ (red line with triangular marks); and effective $\mu$ : measurements (black line with rounded marks) HFSS ${ }^{\mathrm{TM}}$ (orange line with diamond-shaped marks).

without increasing the cell size.

\section{REFERENCES}

[1] R. Marqués, F. Martín, and M. Sorolla, Metamaterials with Negative Parameters: Theory, Design and Microwave Applications. John Wiley \& Sons, 2008.

[2] A. L. Borja, J. Carbonell, V. E. Boria, and D. Lippens, "Highly selective left-handed transmission line loaded with split ring resonators and wires," Applied Physics Letters, vol. 94, no. 14, 2009.

[3] I. Gil, J. Bonache, J. Garcia-Garcia, F. Falcone, and F. Martin, "Metamaterials in microstrip technology for filter applications," in IEEE Antennas and Propagation Society International Symposium, 2005, vol. 1A, July 2005, pp. $668-671$ Vol. 1A.

[4] M. Gil, J. Bonache, I. Gil, J. García-García, and F. Martín, "On the transmission properties of left-handed microstrip lines implemented by complementary split rings resonators," International Journal of Numerical Modelling: Electronic Networks, Devices and Fields, vol. 19, no. 1, pp. $87-103,2006$.

[5] M. Gil, J. Bonache, J. Garcia-Garcia, J. Martel, and F. Martin, "Composite right/left-handed metamaterial transmission lines based on complementary split-rings resonators and their applications to very wideband and compact filter design," IEEE Transactions on Microwave Theory and Techniques, vol. 55, no. 6, pp. $1296-1304,2007$.

[6] M. Gil, J. Bonache, and F. Martin, "Synthesis and applications of new left handed microstrip lines with complementary split-ring resonators etched on the signal strip," IET Microwaves, Antennas Propagation, vol. 2, no. 4, pp. $324-330$, June 2008.

[7] M. Duran-Sindreu, A. Velez, F. Aznar, G. Siso, J. Bonache, and F. Martin, "Applications of open split ring resonators and open complementary split ring resonators to the synthesis of artificial transmission lines and microwave passive components," IEEE Transactions on Microwave Theory and Techniques, vol. 57, no. 12, pp. 3395 -3403, Dec. 2009. 
[8] C. Caloz and T. Itoh, "Application of the transmission line theory of left-handed (LH) materials to the realization of a microstrip "LH line"," in IEEE Antennas and Propagation Society International Symposium, 2002, vol. 2, 2002, pp. $412-415$ vol.2.

[9] A. Grbic and G. V. Eleftheriades, "Experimental verification of backward-wave radiation from a negative refractive index metamaterial," Journal of Applied Physics, vol. 92, no. 10, pp. 5930 -5935, Nov. 2002.

[10] A. Sanada, C. Caloz, and T. Itoh, "Characteristics of the composite right/left-handed transmission lines," Microwave and Wireless Components Letters, IEEE, vol. 14, no. 2, pp. 68 - 70, Feb. 2004.

[11] A. Borja, A. Belenguer, J. Cascon, H. Esteban, and V. Boria, "Wideband passband transmission line based on metamaterial-inspired CPW balanced cells," IEEE Antennas and Wireless Propagation Letters, vol. 10, no. 12, pp. $1421-1424$, Dec. 2011.

[12] C. Caloz, "Dual composite right/left-handed (d-crlh) transmission line metamaterial," IEEE Microwave and Wireless Components Letters, vol. 16 , no. 11 , pp. $585-587$, Nov. 2006.

[13] Y.-H. Ryu, J.-H. Park, J.-H. Lee, J.-Y. Kim, and H.-S. Tae, "DGS dual composite right/left-handed transmission line," IEEE Microwave and Wireless Components Letters, vol. 18, no. 7, pp. 434 -436, July 2008.

[14] W. Tong, Z. Hu, H. Zhang, C. Caloz, and A. Rennings, "Study and realisation of dual-composite right/left-handed coplanar waveguide metamaterial in MMIC technology," IET Microwaves, Antennas Propagation, vol. 2, no. 7, pp. $731-736$, Oct. 2008.

[15] V. Gonzalez-Posadas, J. Jimenez-Martin, A. Parra-Cerrada, L. GarciaMunoz, and D. Segovia-Vargas, "Dual-composite right-left-handed transmission lines for the design of compact diplexers," IET Microwaves, Antennas Propagation, vol. 4, no. 8, pp. 982 -990, Aug. 2010.

[16] C.-Y. Liu, Q.-X. Chu, and J.-Q. Huang, "A planar D-CRLH transmission line structure and its application to leaky-wave antenna," in 9th International Symposium on Antennas, Propagation, and EM Theory (ISAPE), Dec. 2010, pp. $345-348$.

[17] L. Rogla, J. Carbonell, and V. Boria, "Study of equivalent circuits for open-ring and split-ring resonators in coplanar waveguide technology," Microwaves, Antennas Propagation, IET, vol. 1, no. 1, pp. $170-176$, Feb. 2007.

[18] F. Aznar, J. Bonache, and F. Martin, "Improved circuit model for lefthanded lines loaded with split ring resonators," Applied Physics Letters, vol. 92, no. 4, pp. 043 512-1 - 043 512-3, Jan. 2008.

[19] B. C. Wadell, Transmission Line Design Handbook. Artech House, 1991.

[20] C. Caloz and T. Itoh, Electromagnetic Metamaterials: Transmission Line Theory and Microwave Applications. Wiley, 2005.

[21] M. Goldfarb and R. Pucel, "Modeling via hole grounds in microstrip," IEEE Microwave and Guided Wave Letters, vol. 1, no. 6, pp. 135 -137, June 1991.

[22] A. L. Borja, J. Carbonell, V. E. Boria, J. Cascón., and D. Lippens, "A $2 \%$ bandwidth c-band filter using cascaded split ring resonators," IEEE Antennas and Wireless Propagation Letters, vol. 9, pp. 256 -259, 2010.

[23] D. R. Smith, D. C. Vier, T. Koschny, and C. M. Soukoulis, "Electromagnetic parameter retrieval from inhomogeneous metamaterials," Physical Review E, vol. 71, no. 1, p. 036617, 2005.

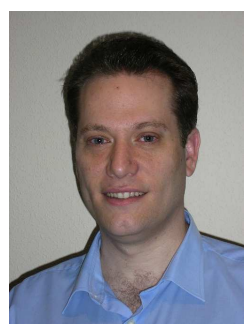

Ángel Belenguer Martínez (M'04) received the telecommunications engineering and Ph.D. degrees from the Universidad Politécnica de Valencia (UPV), Spain, in 2000 and 2009, respectively. He joined the Universidad de Castilla-La Mancha in 2000, where he is now Profesor Titular de Universidad in the Departamento de Ingenieria Electrica, Electronica, Automatica y Comunicaciones. His research interests include methods in the frequency domain for the full-wave analysis of open-space and guided multiple scattering problems, the application of accelerated solving strategies to new problems or structures, EM metamaterials, and Substrate Integrated Waveguide (SIW) devices and their applications.

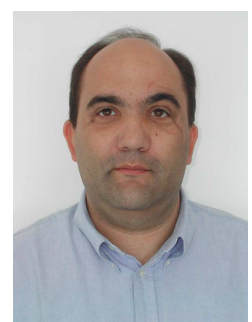

Joaquín Cascón was born in Cuenca, Spain, in 1966. He received his degree in Telecommunications engineering from the Universidad Politécnica de Madrid (UPM), Spain, in 1991, and PhD from the Universidad Politécnica de Valencia, (UPV) Spain, in 1999. From 1991 to 1999 he was with the Optical Communication Group, UPV, where he worked in Optical Communications. In 1999 he joined the Departamento de Ingeniería Eléctrica, Electrónica, Automática y Comunicaciones, Universidad de Castilla-La Mancha (Spain), he is now Catedrático de Escuela Universitaria . His current research interest include EM metamaterials, antennas, tunable structures and their applications in microwave and millimeter-wave technologies.

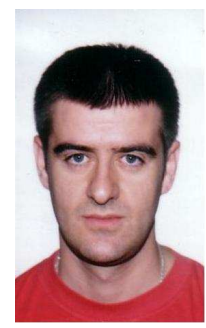

Alejandro L. Borja (M'08) received the M.Sc degree in Telecommunication Engineering and $\mathrm{PhD}$ from the Universidad Politécnica de Valencia, Spain, in 2004 and 2009, respectively. From 2005 to 2006, he was with the University of Birmingham, where he was involved with the development of metamaterial based antennas. He then joined, from 2007 to 2008, the Université de Lille 1, his research activity included the design of metamaterial based filtering structures. Since 2009, he is with the Universidad de Castilla-La Mancha, where he is an Assistant Lecturer. His current research interests include EM metamaterials, UWB antennas, Substrate Integrate Waveguide (SIW) devices and their applications.

Dr. Borja was the recipient of the 2008 CST short paper award.

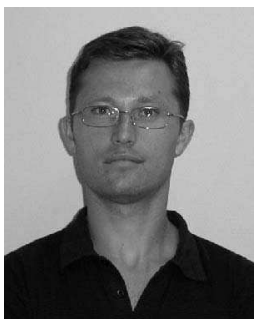

Héctor Esteban González (M’94) received a degree in telecommunications engineering from the Universidad Politécnica de Valencia (UPV), Spain, in 1996, and a Ph.D. degree in 2002. He worked with the Joint Research Centre, European Commission, Ispra, Italy. In 1997, he was with the European Topic Centre on Soil (European Environment Agency). He rejoined the UPV in 1998. His research interests include methods for the full- wave analysis of open-space and guided multiple scattering problems, $\mathrm{CAD}$ design of microwave devices, electromagnetic characterization of dielectric and magnetic bodies, and the acceleration of electromagnetic analysis methods using the wavelets and the fast multipole method.

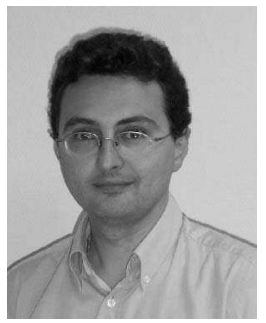

Vicente E. Boria Esbert (S'91-A'99-SM'02) received the Ingeniero de Telecomunicación and the Doctor Ingeniero de Telecomunicación degrees from the Universidad Politécnica de Valencia, Spain, in 1993 and 1997. In 1993 he joined the Universidad Politécnica de Valencia where he is Full Professor since 2003. In 1995 and 1996 he was held a Spanish Trainee position with the European Space research and Technology Centre (ESTEC)-European Space Agency (ESA). He has served on the Editorial Boards of the IEEE Transactions on Microwave Theory and Techniques. His current research interests include numerical methods for the analysis of waveguide and scattering structures, automated design of waveguide components, radiating systems, measurement techniques, and power effects in passive waveguide systems. 